\title{
CRISPR-LRS for mapping transgenes in the mouse genome
}

4 W. Bart Bryant*, Allison Yang, Susan Griffin, Wei Zhang, Xiaochun Long, and Joseph M. Miano*

5 Department of Medicine and Vascular Biology Center. Medical College of Georgia at Augusta

University; Augusta, GA 30912

* corresponding authors (WILBRYANT@augusta.edu and jmiano@augusta.edu)

\section{Abstract}

Microinjected transgenes, including bacterial artificial chromosomes (BACs), insert randomly in the mouse genome. Traditional methods of mapping a transgene are challenging, thus complicating breeding strategies and the accurate interpretation of phenotypes, particularly when a transgene disrupts critical coding or noncoding sequences. Here, we introduce CRISPR-Cas9 long-read sequencing (CRISPR-LRS) to ascertain transgene integration locus and estimated copy number. This method revealed integration loci for both a BAC and Credriver line, and estimated the copy numbers for two other BAC mouse lines. CRISPR-LRS offers an easy approach to establish robust breeding practices and accurate phenotyping of most any transgenic mouse line. 
Despite the routine use of transgenic mice, greater than $90 \%$ of transgenic alleles in the Mouse Genome Database have yet to be mapped $(1,2)$ even though it is broadly accepted that random integration of a transgene can disrupt coding exons and functional noncoding sequences (eg, enhancer, long noncoding RNA), thus complicating data interpretation. Fluorescence in situ hybridization (FISH) can map a transgene to a chromosomal band, but

32 lacks nucleotide resolution (3). Hybridization, targeted locus amplification (TLA), and linear 33 amplification-mediated PCR (LAM-PCR), rely on short-read sequencing and cannot resolve 34 complex genome inversions/deletions (4-7). Inverse PCR has high failure rates due to concatemerization of most transgenes (8). Recently, whole genome sequencing (WGS) with long-read sequencing platforms, PacBio or Oxford Nanopore Technologies (ONT), have been used to map transgenes $(2,9)$. However, even with $\sim 7.5 \mathrm{~Gb}$ of sequencing data for $\sim 3 \mathrm{x}$ coverage of the mouse genome, researchers cannot be assured to find reads that identify the breakpoint of the transgene.

We propose a more targeted approach to map a transgene by combining (i) the RNA programmable CRISPR-Cas9 system (10) and (ii) long-read length coverage of the ONT sequencing platform $(11,12)$. Previous studies have combined CRISPR-Cas9 with LRS to enrich for genomic elements (13-20); however, no studies until now have combined CRISPR and LRS to map transgenes in an animal model. CRISPR-LRS can be designed (i) to target a genomic section (Targeted-CRISPR-LRS) or (ii) to enrich genomic sections (EnrichmentCRISPR-LRS). In this study, CRISPR-LRS successfully mapped a single-copy BAC and a multicopy Cre in the mouse genome. CRISPR-LRS represents a facile tool for mapping transgenes in experimental animal models, thus informing investigators as to best breeding practices and potential genetic confounders.

\section{Results/Discussion}

For the four transgenic mouse lines in this study, seven CRISPR-LRS libraries (Targeted or Enrichment) were sequenced with the minION platform for a total of $\sim 1.8 \mathrm{~Gb}$ at $\sim 400,000$

55 reads (see Supp. Table 1 and 2). Reads mapped to their corresponding reference sequence 56 with a range of $0.02 \%-0.52 \%$ (Supp. Table 2 ) and will be referred to as informative long-reads 57 below. 


\section{CRISPR-LRS mapping of BAC mouse lines}

The mouse line carrying the RP11-744N12 BAC was generated to study human-specific long noncoding RNA, SENCR (21). To map where this $217 \mathrm{~kb}$ BAC integrated into the mouse genome, two Targeted-CRISPR-LRS libraries were made $5 \mathrm{~kb}$ or $3 \mathrm{~kb}$ from the 5' and 3' ends of each BAC sequence, respectively (Figure 1A, Supp. Fig 1i, and Supp. Table 1). Both libraries yielded $0.03 \%$ of reads $(0.9 \mathrm{~kb}-25 \mathrm{~kb})$ that mapped to the reference sequence (Figure $1 \mathrm{Ai}$ and Supp. Table 2). Notably, manual inspection of informative long-reads revealed $\sim 7 \mathrm{~kb}$ of pBACe3.6 vector sequence accompanying transgene and mouse chromosome 15 sequences (Figure 1Ai). Further, informative long-reads over $6 \mathrm{~kb}$ for 5 ' end and $10 \mathrm{~kb}$ for 3 ' end, found RP11-744N12 integrated within the first intron of Egflam (Chr15:7,344,678; GRCm38/mm10) (Figure 1A). CRISPR-LRS also mapped mouse BAC lines, CTD-2518N7 and RP11-997L11, and found informative long-reads with the BAC-cloning vector flanked by 5' and 3' human sequence, indicating integration of at least two copies of each transgenic line (Supp. Fig 1ii and Supp. Fig 2).

While Targeted-CRISPR-LRS mapped the RP11-744N12 BAC integration locus, only a few long-reads covered the integration loci. Enrichment-CRISPR-LRS queried $7.7 \mathrm{~kb}$ and 10.0 $\mathrm{kb}$ for the 5' and 3' terminal ends, respectively, with crRNAs enriching for (i) mouse chromosome 15, (ii) pBACe3.6 cloning vector, and (iii) RP11-744N12 sequences (Figure 1Aii and Supp. Fig 1iv). With 5- and 12- fold enrichment of informative reads at the 5'- and 3'terminal ends, respectively (compare Figure 1Ai to 1Aii), Enrichment-CRISPR-LRS validated the Targeted-CRISPR-LRS mapping data (Figure 1Aii). Further, Sanger sequencing confirmed the breakpoint of RP11-744N12 BAC and chromosome 15, within the first intron of Egflam (Figure 1 Aiii and Supp. Table 1

Genotyping pups from a (+/tg) x (+/tg) cross with primers spanning (i) transgene and chromosome 15 breakpoint and (ii) wild type chromosome 15 loci (Figure 1B), revealed near Mendelian ratio: (+/tg), 17/41 at 41\%; (+/+), 9/41 at 22\%; $(\operatorname{tg} / \mathrm{tg}), 15 / 41$ at $37 \%$. Notably, the BAC transgene exists as a single copy with no loss of mouse genomic sequence at the site of integration and healthy, homozygous transgenic mice indicate the absence of overt pathology.

While CRISPR-LRS mapped RP11-744N12 as a single copy, we wanted to check for the possibility of additional integration sites as one study reported multiple integration loci for $\sim 10 \%$

91 of their EGFP-reporter lines by FISH (3). To verify a single integration locus, heterozygous $(+/ \mathrm{tg})$

92 pups were crossed and progeny genotypes were assessed with BAC-specific primers, (Figure

93 1B). As expected, BAC-specific amplicons were present in (+/tg) and (tg/tg) pups (Figure 1B).

94 However, if a wild type (+/+) pup from the heterozygous cross exhibited BAC-specific 
amplicons, then this result would indicate that CRISPR-LRS missed additional transgene integration sites. Notably, wild type (+/+) pups from the $(+/ \mathrm{tg}) \times(+/ \mathrm{tg})$ did not exhibit BACspecific amplicon products (Figure 1B), demonstrating CRISPR-LRS did successfully map the RP11-744N12 BAC as a single copy transgenic mouse line.

Fortuitously, as all BAC transgenes were flanked with BAC-cloning vector sequence (Figure 1 and Supp. Fig 2), copy number quantification was possible. qPCR, routine for copy number variation analysis $(1,2,5,9)$, targeted the chloramphenicol resistance gene, a common gene within BAC-vectors. With a single integration (Figure 1), (+/tg) and (tg/tg) RP11744N12 pups were queried by qPCR finding one and two BAC transgene copies, respectively (Supp. Fig $3 A)$. As the other BAC-lines exhibited tandem integrations, only (+/tg) pups were queried, with qPCR showing $~ 2-3$ copies for both lines. Collectively, qPCR was consistent with the CRISPRLRS mapping data (Figure 1, Supp. Fig1 i and ii, Supp. Fig 2, and Supp. Fig 3A).

\section{CRISPR-LRS mapping of Cre-driver mouse line}

Most small transgenes lack a mapped integration locus and can possess dozens of copies of the transgene, as well as complex rearrangements of the host genome $(1,2,22)$. We next applied CRISPR-LRS to map Sm22-Cre, a mouse line used to excise floxed DNA sequences in early embryonic heart and smooth muscle cell-containing tissues (23). To map the BAC lines, CRISPR-LRS targeted at least $2 \mathrm{~kb}$ from terminal ends of the transgene (Figure 1, Supp. Fig 1i-ii, and Supp. Fig 2). However, because Cre is significantly smaller than a BAC, a different Targeted-CRISPR-LRS approach was used. Specifically, two independent libraries targeting $0.8 \mathrm{~kb}$ or $0.5 \mathrm{~kb}$ from the 5' and 3' end, respectively, were run on one flow cell (Figure $2 \mathrm{Ai}$, Supp. Fig 1iii, and Supp. Table 1). At $0.52 \%$, the Sm22-Cre libraries contained more informative long-reads over the BAC libraries (Figure 1, Figure 2Ai, Supp. Fig 2, and Supp. Table 2). Manual interrogation and alignment of $>6 \mathrm{~kb}$ informative long-reads elucidated a minitiled Cre transgene integration map consisting of multiple copies of $\mathrm{Cre}$ and genomic inversions (Figure 2Ai). Three informative long-reads revealed a breakpoint between one of the Cre transgenes and 91,527,881 bp on chromosome 14 (GRCm38/mm10) (Figure 2Ai, dashed black line box). Sanger sequencing verified the Cre and host chromosome breakpoint (Figure 2Aii and Supp. Table 1).

Genotyping pups from a $(+/ \mathrm{tg}) \times(+/ \mathrm{tg})$ cross with primers spanning (i) the breakpoint of Cre and chromosome 14 and (ii) wild type chromosome 14 loci (Figure 2B), revealed near Mendelian ratio: (+/tg), 20/41 at $49 \% ;(+/+), 8 / 41$ at $19 \% ;(\operatorname{tg} / \mathrm{tg}), 13 / 41$ at $32 \%$. 
To check for additional integration loci for the Cre-driver, heterozygous pups were crossed and progeny genotypes assessed with Cre-specific primers, D and E (Figure 2B). Both

$130(+/ \mathrm{tg})$ and $(\mathrm{tg} / \mathrm{tg})$ pups yielded amplicon products for the internal Cre primers, as expected

131 (Figure 2B). Notably, wild type pups from the $(+/ \mathrm{tg}) \times(+/ \mathrm{tg})$ cross did not yield Cre-specific

132 amplicon products (Figure $2 \mathrm{~B}$ ), demonstrating CRISPR-LRS successfully mapped one

133 integration locus for the Sm22Cre mouse line.

134 As small transgenes typically integrate as concatemers (1,2), our mini-tiled integration

135 map for Sm22Cre could not firmly establish copy number (Figure 2A). To address this limitation,

136 qPCR determined the copy number for the line with $\sim 20$ and $\sim 40$ copies of Cre for (+/tg) and

137 (tg/tg) pups, respectively (Figure 2Aiii and Supp. Figure 3B). As the Sm22Cre mouse has 20

138 copies of Cre per allele, there were more Cas9-ribonucleoprotein (RNP) targets to cleave

139 compared to the BAC lines, explaining how the Sm22Cre-CRISPR-LRS libraries contained

140 more informative mapped long-reads over the BAC-CRISPR-LRS libraries (Figure 1, Figure 2,

141 Supp. Fig 2, Supp. Fig 3, and Supp. Table 2).

\section{Conclusions}

There are several benefits of mapping transgenes in animal models such as mice. First, 146 mapping allows investigators to accelerate the generation of desired outcomes in a complex

147 breeding scheme, (e.g., floxed alleles with Cre-driver lines). Second, it enables quality design of 148 genotyping assays for colony maintenance and zygosity determination. Lastly, and arguably 149 most important, it alerts one to confounding genetics caused by insertion of the transgene in a 150 coding/noncoding sequence or regulatory element $(1,2,5,9,22)$.

Of the available strategies to map transgenes, WGS $(2,9)$ and $\operatorname{TLA}(1,5)$ have the most

152 traction. However with WGS, most of the sequence data is uninformative and sequence depth is 153 substantial with $\sim 2-5 x$ coverage of the host genome $(2,9)$. Here, with only $\sim 1.8 \mathrm{~Gb}$ of cumulative 154 sequence data, CRISPR-LRS mapped four mouse lines with two lines yielding PCR validated 155 chromosome coordinates. TLA can be technically challenging for most labs as (i) it has

156 numerous steps; crosslinking, fragmentation, re-ligation, and amplification, and (ii) analysis can

157 require extensive computational expertise due to the nature of these fragmented sequence

158 libraries (1, 5). CRISPR-LRS (i) has less steps; Cas9 cleavage and adaptor ligation, and (ii) only 159 requires mapping long-reads with an open source tool, minimap2 (24). We envision CRISPR-

160 LRS as the 'go-to' method of mapping transgenes in any organism with a reference genome. 


\section{Methods}

\section{Transgenic mice}

164 The SENCR BAC and Sm22Cre mouse lines were reported previously (21), (23). The human CTD-2518N7 and RP11-997L11 BAC transgenic lines were generated by Cyagen (www.cyagen.com) using strain C57BL/6J. All mice were maintained on strain C57BL/6J through repeated back-crossing, refreshing the breeders every 5 generations to mitigate genetic drift. Mouse experiments were approved by Medical College of Georgia at Augusta University Institutional Animal Care and Use Committee (approval numbers 2019-1000 and 2019-0999).

\section{Long-read library preparation}

172 Genomic DNA (gDNA) was isolated from mouse liver tissue using Qiagen DNeasy Blood \&

173 Tissue Kit (cat\#69504) following manufacturer's instructions (www.qiagen.com). To limit

174 shearing of gDNA, wide bore pipette tips were used. Libraries ( $5 \mu \mathrm{g} g \mathrm{gNA})$ were prepared for

175 four different transgenic mouse lines following manufacturer's instructions for Cas 9 sequencing

176 kit (SQK-CS9109) using the long fragment buffer option during library prep clean-up for seven

177 total CRISPR-LRS libraries (www.nanoporetech.com). crRNAs were designed using

178 CHOPCHOP (25) with default parameters (Supp. Table I) (https://chopchop.cbu.uib.no).

179 Following suggestions from ONT, all crRNAs, tracrRNA, and HiFi Cas9 were ordered from IDT

180 (www.idt.dna.com). To ensure adequate read length needed to accurately map transgene

181 integration loci, crRNAs were designed to target within $5 \mathrm{~kb}$ of the terminal 5' and 3' ends of the

182 BAC sequence. Further, a Targeted- or an Enrichment- CRISPR-LRS approach was performed 183 for each mouse line (see Supp. Fig. 1 for flow chart). For Targeted-CRISPR-LRS, crRNAs were designed at the 5' and 3' ends of the transgene where RNPs were loaded with either one or multiple crRNAs (Supp. Figure 1i - iii and Supp. Table 1). For Enrichment-CRISPR-LRS, tandem crRNAs were designed up- and down- stream of the genomic region of interest (ROI)

187 and loaded onto one RNP $(14,20)$ (Supp. Figure 1iv and Supp. Table 1). For both approaches, 188 pre-existing DNA ends were dephosphorylated before Cas9-cutting, which yielded preferential 189 ligation of nanopore adaptors to fresh Cas9 cleavage sites as a means to target specific 190 genomic ROls. For the RP11-744N12 BAC mouse line, two crRNAs, one targeting the 5' and 191 one targeting the 3' end of the BAC sequence, were loaded onto two separate RNPs for two 192 independent libraries (Supp. Fig. 1i). Since an integration locus for the RP11-744N12 BAC 193 mouse line was determined, Enrichment-CRISPR-LRS was further performed following 
manufacturer's instructions (SQK-CS9109) (www.nanoporetech.com). Both 5' and 3' integration loci were probed with four crRNAs loaded onto one RNP (Supp. Fig. 1iv). For the remaining BAC mouse lines, CTD-2518N7 and RP11-997L11, two crRNAs were loaded onto one RNP for one Cas9 library run (Supp. Fig. 1ii). For the Cre-driver mouse line, overlapping crRNAs targeting Cre were designed at least $0.5 \mathrm{~kb}$ from 5' or 3' end with two crRNAs loaded onto two separate RNPs and the two independent libraries combined on one flow cell (Supp. Fig. 1iii).

\section{Nanopore sequencing and data analysis}

202 Cas9 targeted long-read libraries were run on R9.4.1 flow cells on a minION Mk 1B following

203 manufacturer's instructions for Cas9 sequencing kit (SQK-CS9109) (www.nanoporetech.com).

204 Reads were converted from fast5 to fastq with guppy (v4.2.2) on MinKNOW (v20.10.3)

205 MinKNOW Core (v4.1.2) with fast base-calling option for the base-call model and minimum Q-

206 score of 7 option for read filtering. To analyze LRS results, guppy base-called fastq files were

207 imported into Qiagen CLC Genomics Workbench (www.qiagen.com). Reference sequences

208 specific to each transgenic mouse line were obtained from NCBI nucleotide database

209 (www.ncbi.nlm.nih.gov/nucleotide/) and alignments generated using the Long-Read Support

210 (beta) plugin available in Qiagen CLC Genomics Workbench (www.digitalinsights.qiagen.com),

211 which utilizes components of open-source tool minimap2 (24). Default parameters for the long-

212 read alignment plugin were used. Aligned informative long-reads were extracted and manually

213 queried against NCBI nr/nt and refseq genome databases

214 (https://blast.ncbi.nlm.nih.gov/Blast.cgi) and UCSC genome browser with BLAT tool

215 (https://genome.ucsc.edu). Graphical output obtained from CLC Genomics Workbench and

216 GraphPad (www.graphpad.com) were amended with Adobe Illustrator (www.adobe.com)

217 (Adobe Systems, San Jose, CA, USA) for illustration purposes.

219 Genotyping and transgene copy number analysis

220 Small ear biopsies were taken before weaning and gDNA was extracted using Qiagen DNeasy

221 Blood \& Tissue Kit (cat\#69504) following manufacturer's instructions (www.qiagen.com).

222 Progeny of $(+/ \mathrm{tg}) \times(+/ \mathrm{tg})$ heterozygous crosses were assessed for CRISPR-LRS mapped

223 integration loci of the transgene. For genotyping, PCR conditions were the following: step 1, 95

$224{ }^{\circ} \mathrm{C}$ for $3 \mathrm{~min}$; step $2,95^{\circ} \mathrm{C}$ for $30 \mathrm{sec}, 58^{\circ} \mathrm{C}$ for $30 \mathrm{sec}$, and $72^{\circ} \mathrm{C}$ for 1 min for 35 cycles; step

$2253,72{ }^{\circ} \mathrm{C}$ for $10 \mathrm{~min}$. Sequences for all genotyping amplicons were confirmed by Sanger

226 sequencing (Supp. Table I). For transgene copy number determination, gDNA was diluted to

22750 ng for input. For the BAC mouse lines, two primer sets to the chloramphenicol resistance 
228 gene served as proxy for the BAC transgene, where values were normalized to an internal

229 control locus (Supp. Table 1) (1, 2, 9). For the Cre-driver mouse line, two primer sets to Cre

230 were normalized to same internal control locus used for the BAC mouse lines. The Itga8-

231 CreER $R^{T 2}$ mouse, known to have one copy of Cre (manuscript in preparation), served as a

232 calibrator for one copy of Cre. Real time quantitative PCR conditions were the following: step1,

$23395^{\circ} \mathrm{C}$ for $3 \mathrm{~min}$; step $2,95^{\circ} \mathrm{C}$ for $30 \mathrm{sec}, 60^{\circ} \mathrm{C}$ for $30 \mathrm{sec}$, and $72^{\circ} \mathrm{C}$ for $30 \mathrm{sec}$ for 40 cycles.

\section{Data availability}

236 Data generated by ONT LRS have been submitted to NCBI SRA database

237 (www.ncbi.nlm.nih.gov/sra) under BioProject number PRJNA759232 and will be publically

238 available after manuscript acceptance. 
241 Declarations

242 Ethics approval and consent to participate

243 Not applicable

244

245 Consent for publication

246 Not applicable

247

248 Availability of data and materials

249 Nanopore long read sequencing data are available at NCBI Sequence Read Archive (SRA)

250 under accession number PRJNA759232.

251

252 Competing interests

253 The authors declare no competing interests

254

255 Funding

256 Work was supported by grants HL138987 and HL147476 to J.M.M. and HL122686 and

257 HL139794 to X.L.

258

259 Author contributions

260 W.B.B. and J.M.M designed the study.

261 A.Y., S.G. and WZ maintained mouse colonies.

262 W.B.B. performed the experiments.

263 W.B.B. and J.M.M. analyzed and interpreted data

264 X.L. provided liver tissue.

265 W.B.B. and J.M.M. wrote the paper.

266 All authors read and approved the final manuscript.

268 Acknowledgements

269 We wish to thank Akelia Wauchope-Odumbo and Carl Woodham at Oxford Nanopore

270 Technologies (ONT) for their combined help with use of long-read nanopore

271 sequencing. 
1. Goodwin LO, Splinter E, Davis TL, Urban R, He H, Braun RE, et al. Large-scale discovery of mouse transgenic integration sites reveals frequent structural variation and insertional mutagenesis. Genome Res. 2019;29(3):494-505. Characterizing a Transgene Integration Site by Nanopore Sequencing. G3 (Bethesda). 2019;9(5):1481-6. 3. Nakanishi T, Kuroiwa A, Yamada S, Isotani A, Yamashita A, Tairaka A, et al. $\mathrm{FISH}$ analysis of $142 \mathrm{EGFP}$ transgene integration sites into the mouse genome. Genomics. 2002;80(6):564-74.

4. Dubose AJ, Lichtenstein ST, Narisu N, Bonnycastle LL, Swift AJ, Chines PS, et al. Use of microarray hybrid capture and next-generation sequencing to identify the anatomy of a transgene. Nucleic Acids Res. 2013;41(6):e70.

5. Cain-Hom C, Splinter E, van Min M, Simonis M, van de Heijning M, Martinez M, et al. Efficient mapping of transgene integration sites and local structural changes in Cre transgenic mice using targeted locus amplification. Nucleic Acids Res. 2017;45(8):e62. 6. Schmidt M, Schwarzwaelder K, Bartholomae C, Zaoui K, Ball C, Pilz I, et al. High-resolution insertion-site analysis by linear amplification-mediated PCR (LAMPCR). Nat Methods. 2007;4(12):1051-7.

7. de Vree PJ, de Wit E, Yilmaz M, van de Heijning M, Klous P, Verstegen MJ, et al. Targeted sequencing by proximity ligation for comprehensive variant detection and local haplotyping. Nat Biotechnol. 2014;32(10):1019-25.

8. Liang Z, Breman AM, Grimes BR, Rosen ED. Identifying and genotyping transgene integration loci. Transgenic Res. 2008;17(5):979-83.

9. Sailer S, Coassin S, Lackner K, Fischer C, McNeill E, Streiter G, et al. When the genome bluffs: a tandem duplication event during generation of a novel Agmo knockout mouse model fools routine genotyping. Cell Biosci. 2021;11(1):54.

10. Jinek M, Chylinski K, Fonfara I, Hauer M, Doudna JA, Charpentier E. A programmable dual-RNA-guided DNA endonuclease in adaptive bacterial immunity. Science. 2012;337(6096):816-21.

11. Deamer D, Akeson M, Branton D. Three decades of nanopore sequencing. Nat Biotechnol. 2016;34(5):518-24.

12. Kono N, Arakawa K. Nanopore sequencing: Review of potential applications in functional genomics. Dev Growth Differ. 2019;61(5):316-26.

13. Wongsurawat T, Jenjaroenpun P, De Loose A, Alkam D, Ussery DW, Nookaew I, et al. A novel Cas9-targeted long-read assay for simultaneous detection of IDH1/2 mutations and clinically relevant MGMT methylation in fresh biopsies of diffuse glioma. Acta Neuropathol Commun. 2020;8(1):87.

14. Walsh T, Casadei S, Munson KM, Eng M, Mandell JB, Gulsuner S, et al. CRISPR-Cas9/long-read sequencing approach to identify cryptic mutations in BRCA1 and other tumour suppressor genes. J Med Genet. 2020.

15. Miyamoto S, Aoto K, Hiraide T, Nakashima M, Takabayashi S, Saitsu H. Nanopore sequencing reveals a structural alteration of mirror-image duplicated genes in a genome-editing mouse line. Congenit Anom (Kyoto). 2020;60(4):120-5. 
16. Watson CM, Crinnion LA, Hewitt S, Bates J, Robinson R, Carr IM, et al. Cas9based enrichment and single-molecule sequencing for precise characterization of genomic duplications. Lab Invest. 2020;100(1):135-46. 17. van Haasteren J, Munis AM, Gill DR, Hyde SC. Genome-wide integration site detection using Cas9 enriched amplification-free long-range sequencing. Nucleic Acids Res. 2021;49(3):e16. 18. Gabrieli T, Sharim H, Fridman D, Arbib N, Michaeli Y, Ebenstein Y. Selective nanopore sequencing of human BRCA1 by Cas9-assisted targeting of chromosome segments (CATCH). Nucleic Acids Res. 2018;46(14):e87.

19. McDonald TL, Zhou W, Castro CP, Mumm C, Switzenberg JA, Mills RE, et al. Cas9 targeted enrichment of mobile elements using nanopore sequencing. Nat Commun. 2021;12(1):3586. 20. Gilpatrick T, Lee I, Graham JE, Raimondeau E, Bowen R, Heron A, et al. Targeted nanopore sequencing with Cas9-guided adapter ligation. Nat Biotechnol. 2020;38(4):433-8.

21. Lyu Q, Xu S, Lyu Y, Choi M, Christie CK, Slivano OJ, et al. SENCR stabilizes vascular endothelial cell adherens junctions through interaction with CKAP4. Proc Natl Acad Sci U S A. 2019;116(2):546-55.

339 23. Miano JM, Ramanan N, Georger MA, de Mesy Bentley KL, Emerson RL, Balza

$340 \mathrm{RO}$, Jr., et al. Restricted inactivation of serum response factor to the cardiovascular system. Proc Natl Acad Sci U S A. 2004;101(49):17132-7.

24. Li H. Minimap2: pairwise alignment for nucleotide sequences. Bioinformatics. 2018;34(18):3094-100.

25. Labun K, Montague TG, Krause M, Torres Cleuren YN, Tjeldnes H, Valen E. 


\section{Figure Legends}

\section{Figure 1. CRISPR-LRS mapping of RP11-744N12 BAC to chromosome 15 in mouse} genome. (A) Dashed red line represents integration locus of RP11-744N12 BAC within first intron of Egflam on chromosome 15, section qA1. Blue and yellow arrows represent the 5' and 3' end crRNAs, respectively, used to make Targeted-CRISPR-LRS libraries (see Supp. Fig1i flow chart). (i) Histograms for informative reads from Targeted-CRISPR-LRS libraries at either 5' or 3' end of the RP11-744N12 BAC. (ii) Black triangles represent flanking tandem crRNAs used to make Enrichment-CRISPR-LRS libraries to interrogate 5' and 3' ends of the RP11-744N12 integration locus on chromosome 15, section qA1. (iii) Sanger sequencing verification of CRISPR-LRS mapped RP11-744N12 integration locus for 5' and 3' ends of the RP11-744N12 integration locus. (B) PCR genotyping of mice progeny from $(+/ \mathrm{tg}) \times(+/ \mathrm{tg})$ cross with primer schematics. Primer pair A+B interrogated the breakpoint junction of RP11-744N12 BAC and chromosome 15, section $q A 1$. Primer pair $A+C$ interrogated wild type integration locus. To check for presence of RP11-744N12 BAC transgene, internal primer pairs $D+E$ and $F+G$ targeted 5' and 3' regions of the RP11-744N12 BAC sequence, respectively.

Figure 2. CRISPR-LRS mapping of Sm22Cre-driver to chromosome 14 in mouse genome.

(A) Dashed red line represents integration locus of Sm22Cre-driver within chromosome 14, section qE2.1. Informative long reads were compiled to build an integration locus map. Dashed black line box represents Sm22Cre-driver integration locus. Blue and yellow arrows represent internal overlapping 5' and 3' end crRNAs used to make a Targeted-CRISPR-LRS library (see Supp. Fig 1iii flow chart). (i) Histogram for informative reads from the Targeted-CRISPR-LRS

372 library. (ii) Sanger sequencing verification of CRISPR-LRS mapped Sm22Cre-driver integration

373 locus, represented by dashed black line box highlighted in (A). (iii) qPCR determination of

374 transgene copy number with $(+/ \mathrm{tg})$ and $(\mathrm{tg} / \mathrm{tg})$ pups with approximately 20 and 40 copies,

375 respectively. Data normalized to internal control locus and calibrator (Itga8-CreER ${ }^{T 2}$, see

376 Methods for details). $N=2$ for calibrator (Itga8-CreER $\left.{ }^{T 2}\right), N=4$ for Sm22Cre (+ $\left./ \mathrm{tg}\right)$ and $(\mathrm{tg} / \mathrm{tg})$.

377 Values graphed as mean \pm SD. (B) PCR genotyping of mice progeny from $(+/ \mathrm{tg}) \times(+/ \mathrm{tg})$ cross

378 with primer schematics. Primer pair A+B interrogated the breakpoint junction of Sm22Cre-driver

379 and chromosome 14, section qE2.1, represented by dashed black line box highlighted in $(A)$.

380 Primer pair $A+C$ interrogated wild type integration locus. To check for presence of transgene,

381 internal primer pair D+E targeted the Cre transgene. 


\section{Supplemental Figure 1. CRISPR-LRS flow chart.}

Overview of library preparation for Targeted- (i-iii) and Enrichment- (iv) CRISPR-LRS libraries.

\section{Supplemental Figure 2. CRISPR-LRS determination of tandem BAC integration for two}

BAC mouse lines. Overview of Targeted-CRISPR-LRS sequencing illustrating tandem integration of BAC sequence for (A) CTD-2518N7 and (B) RP11-997L11 BAC mouse lines (See Supp. Fig1ii flow chart).

\section{Supplemental Figure 3. Relative transgene copy number for BAC and Cre-driver mouse}

lines. GPCR to determine transgene copy number, normalized to internal control locus. (A) Two primer sets (i and ii) targeting chloramphenicol resistance gene, common gene found in BAC cloning vectors. For RP11-744N12 mouse line, $(+/ \mathrm{tg})$ and $(\mathrm{tg} / \mathrm{tg})$ pups demonstrated one and two copies, respectively. For both CTD-2518N7 and RP11-997L11 mouse lines, $(+/ \mathrm{tg})$ pups demonstrated approximately 3 copies. (B) Two primer sets (i and ii) targeting Cre sequence. Itga8-CreER $R^{T 2}$ mouse served as calibrator for one copy of $\mathrm{Cre}$, serving as $(+/ \mathrm{tg})$ control. For Sm22Cre-driver mouse line, $(+/ \mathrm{tg})$ and $(\mathrm{tg} / \mathrm{tg})$ pups demonstrated approximately 20 and 40 copies, respectively. $n=9$ for RP11-744N12 $(+/ \mathrm{tg}), n=7$ for RP11-744N12 $(\mathrm{tg} / \mathrm{tg}), n=6$ for CTD-2518N7 (+/tg), $n=3$ for RP11-997L11 (+/tg), $n=2$ for Itga8-CreER ${ }^{T 2}, n=4$ for both Sm22Cre $(+/ \mathrm{tg})$ and $(\mathrm{tg} / \mathrm{tg})$. Values graphed as mean \pm SD. 
Bryant et. al, Figure 1

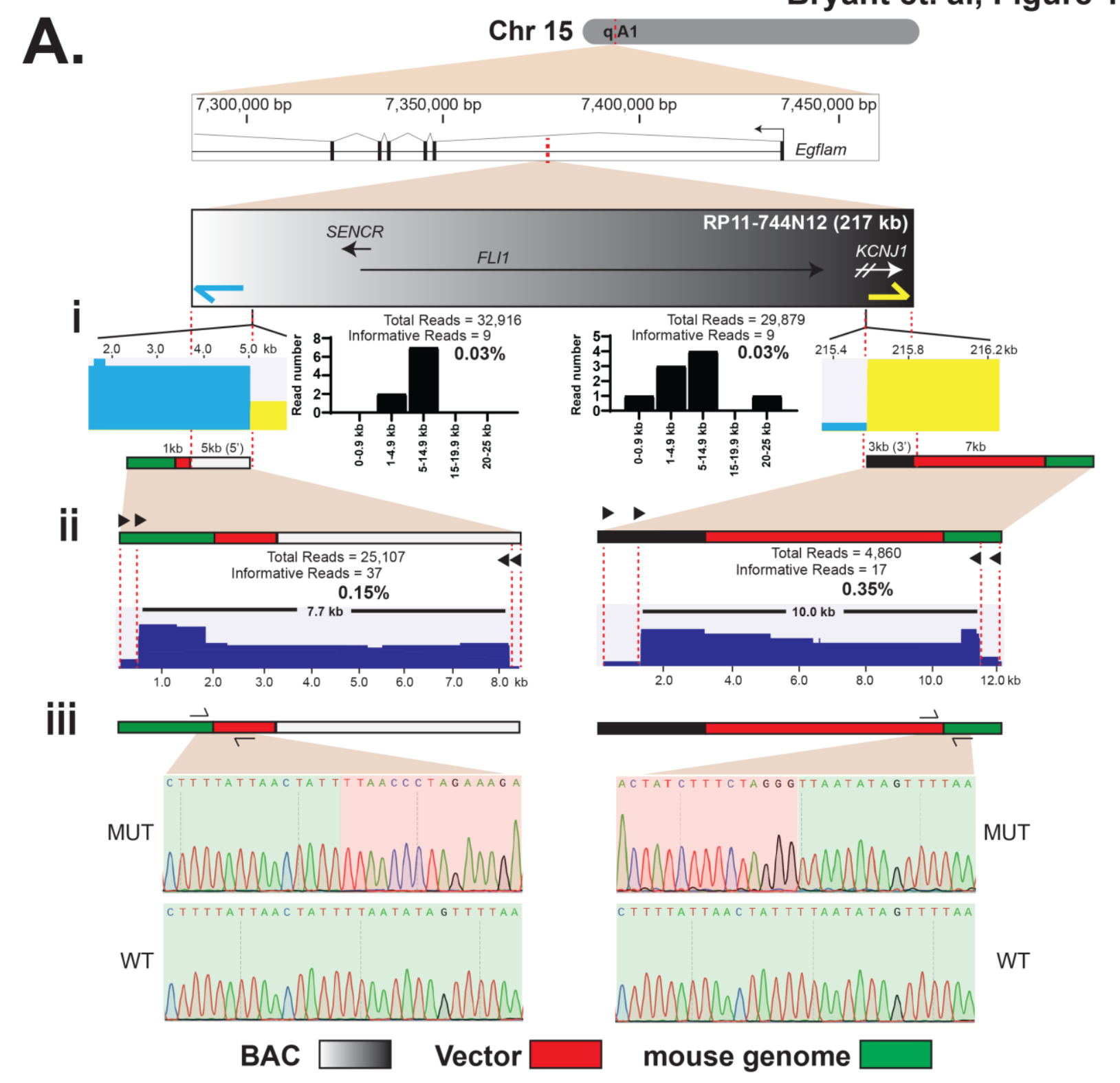

B.

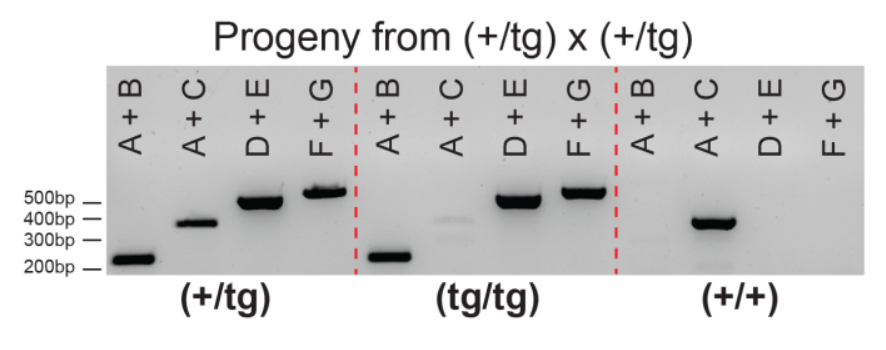

Schematics for primers

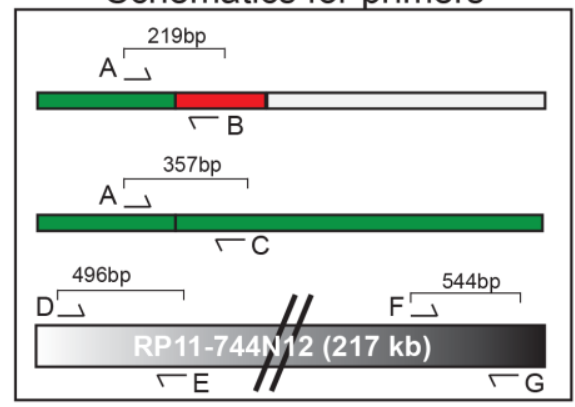




\section{Bryant et. al, Figure 2}

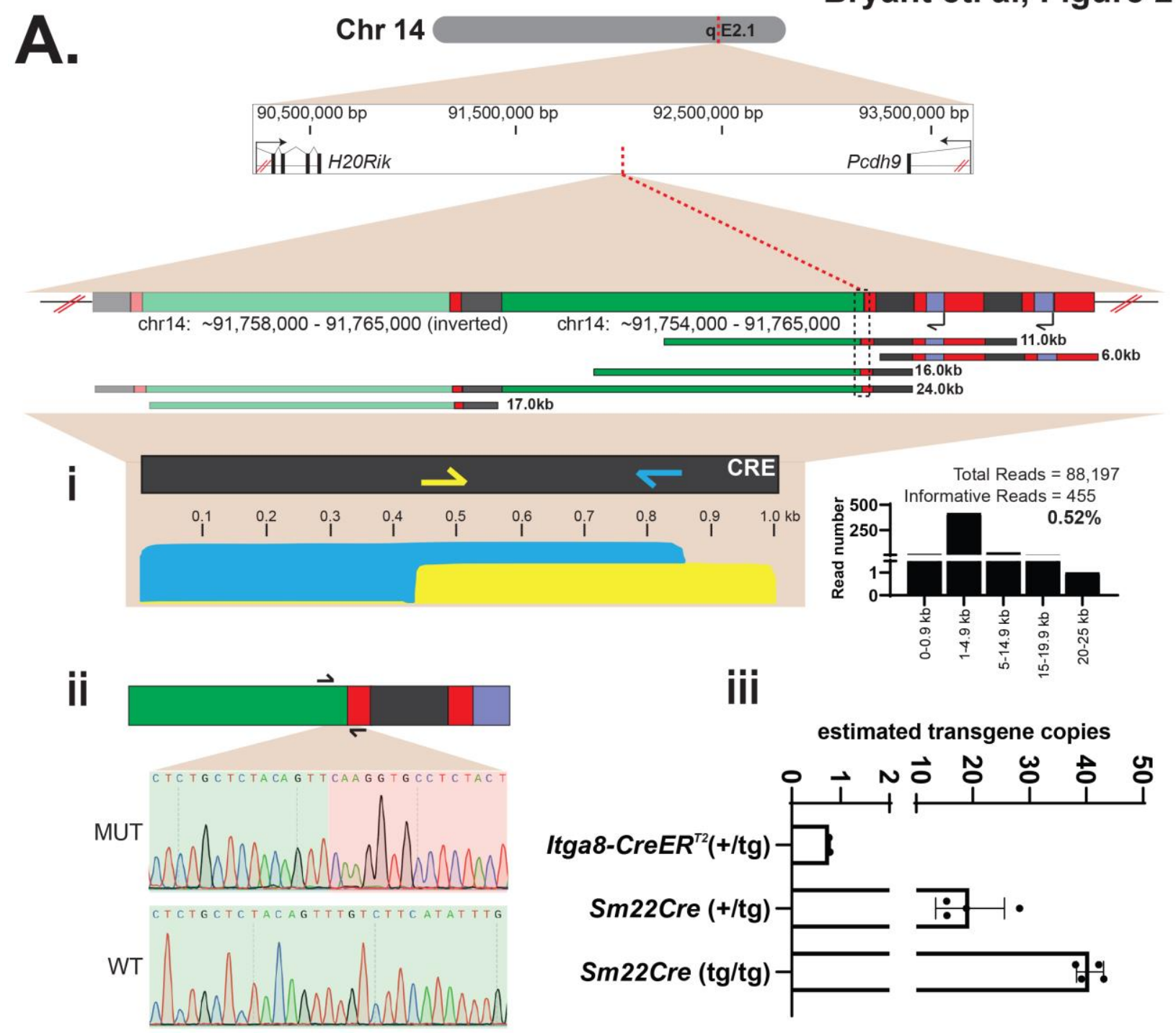

\section{Sm22 promoter $\square$ Vector $\square$ CRE $\square$ mouse genome}

B.

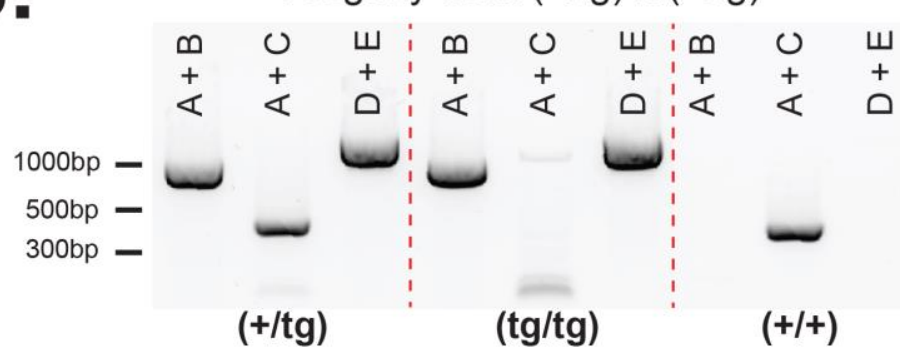

Schematics for primers

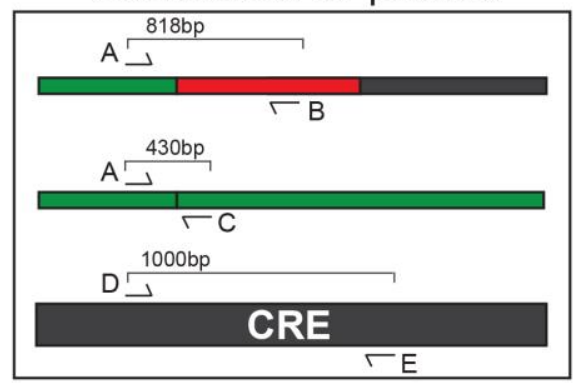


Bryant et. al, Supp. Figure 1

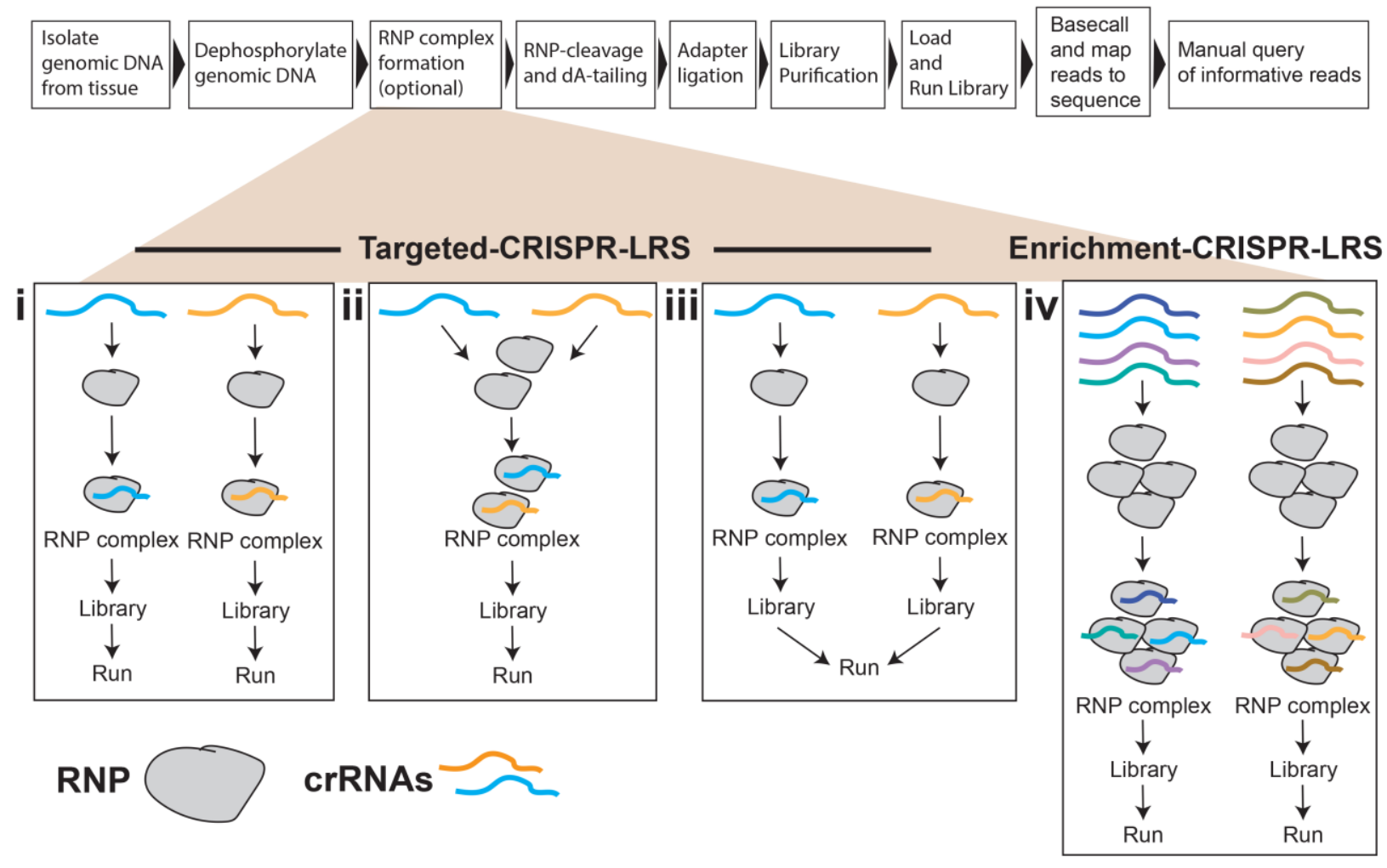



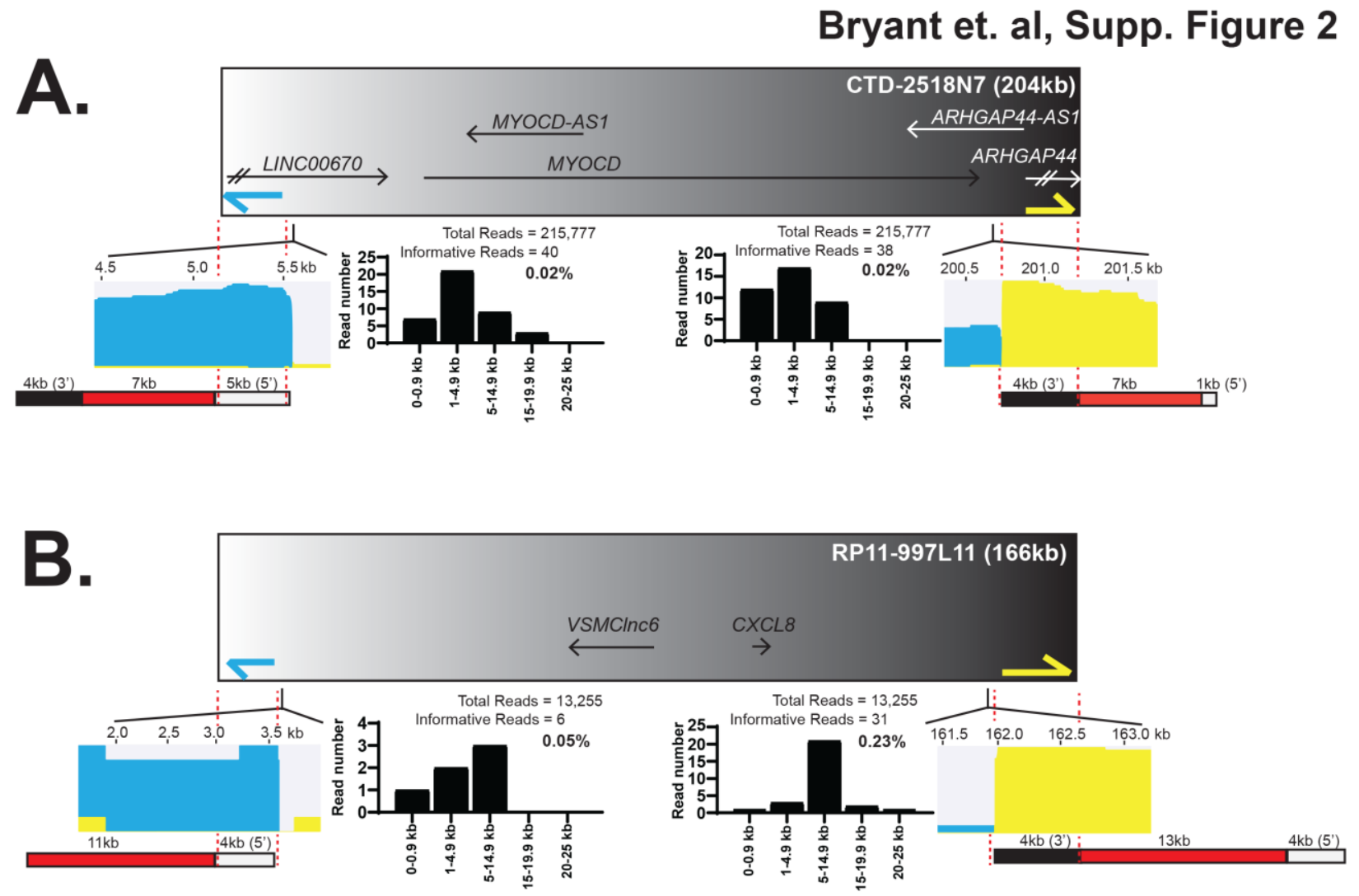

\section{BAC $\square$ Vector}




\section{Bryant et. al, Supp. Figure 3}
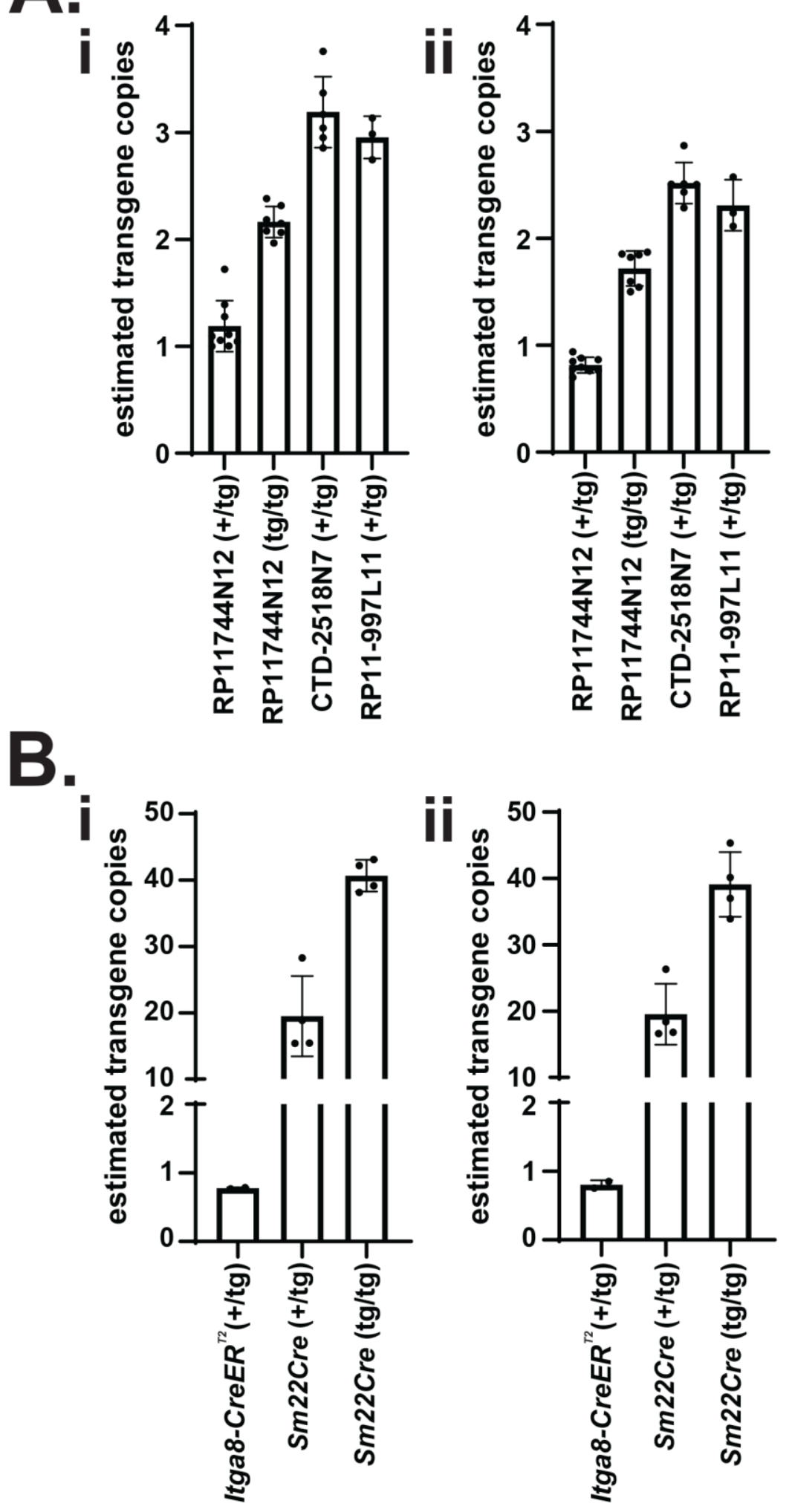